\title{
A Multi-modal Approach to Segmentation of Tubular Structures
}

\author{
Matthias Harders and Gábor Székely \\ Swiss Federal Institute of Technology \\ Communication Technology Laboratory \\ ETH Zentrum, CH-8092 Zürich, Switzerland \\ \{mharders, szekely\}@vision.ee.ethz.ch
}

\begin{abstract}
In this paper we describe a new paradigm for interactive segmentation of medical 3D volume data. We are using a 3D haptic device to enhance the segmentation process with additional sensory feedback. The segmentation of tubular structures in the human body serves as an initial case study for our system. The haptic interaction allows the user to extract the centerline of a tubular structure, even if the image data quality is insufficient. The segmented path is then used to reorient crosssections through the data volume, which helps to extract the boundary of the $3 \mathrm{D}$ object in a subsequent step.
\end{abstract}

\section{Introduction}

The segmentation of 3D medical datasets obtained from a variety of tomographic imaging modalities, such as CT or MRI, can be classified with regard to its degree of automation. In between the two extremes of manual and automatic segmentation lie semi-automated algorithms, which try to merge the advantages of both worlds. Rather than attempting to duplicate the complex and poorly understood human capability to recognize objects, they try to provide an interactive environment in which users control the segmentation process and exploit their expert knowledge. These algorithms can be further classified according to the underlying interaction paradigms in either two or three dimensions, but systems that exploit the possibilities of true interactive 3D segmentation are more seldom [2,43. This is often justified by the problems that arise due to adding another dimension to the user interaction. Editing, controlling and interacting in three dimensions often overwhelms the perceptual powers of a human operator. Moreover, todays desktop metaphors are based on two-dimensional interaction and can not easily be extended to three dimensions. Finally, the visual channel of the human sensory system is not suitable for the perception of volumetric data. In the presented project these limitations are alleviated by enhancing the segmentation process with additional sensory feedback. We focus on providing perceptual information to the medical expert by using a haptic device, which provides 3 DOF translational feedback. We are currently concentrating on the semi-automatic detection and extraction of tubular structures in medical datasets. Usually this research area is subdivided with respect to the different medical fields the examined structures stem from. Nevertheless, several problems can be identified 
which are common to segmentation tasks in all these subfields. Success or failure of reported algorithms is often largely dependent on the quality of the image data. For example, the variable intensity of an unevenly distributed contrast agent inside a vessel might cause artifacts during segmentation. Furthermore, the presence of pathological changes, which is often the case for the objects of interest, can cause problems for template or model based approaches that rely on the size or shape of the contour. Additionally, the handling of junctions in tubular structures often poses a difficulty, that has to be overcome. Also, twodimensional approaches have limitations, because tubular structures often follow a tortuous and curved course through 3D space. This makes the accurate tracing of their geometry an extremely difficult task. Furthermore, tightly folded structures are often sliced at an oblique angle, resulting in extreme deterioration of image quality as tangential slicing direction is approached. These problems may make the slice-by-slice reconstruction of their 3D geometry a very complicated and error prone task.

\section{Multi-modal Segmentation Tool}

In order to overcome the described difficulties, we focus on using force-feedback as an additional channel to mediate information. The initial step of our multimodal approach is the haptically assisted extraction of the centerline of a tubular structure. To do this we adopt an approach similar to the one used by Bartz for enhanced navigation 11. First we create a binarization of our data volume by thresholding. From the resulting dataset $S$ we compute an Euclidean distance: For each $(x, y, z) \in S$, we compute the distance map value

$$
D M(x, y, z)=\min _{\left(x_{i}, y_{i}, z_{i}\right) \in S} d\left[(x, y, z),\left(x_{i}, y_{i}, z_{i}\right)\right],
$$

where $d$ denotes the Euclidean distance from a voxel that is part of the tubular structure to a voxel of the surrounding tissue. In the next step we negate the 3D distance map and approximate the gradients by central differences. Moreover, to ensure the smoothness of the computed forces, we apply a $5 \times 5 \times 5$ binomial filter. This force map is precomputed before the actual interaction, to ensure a stable force-update. Because the force vectors are located at discrete voxel positions, we have to do a tri-linear interpolation to obtain the continuous gradient force map we need during interaction. The computed forces can now be utilized to guide a user on a path close to the centerline of the tubular structure. In the case of good data quality, the user "falls through" the dataset guided along the $3 \mathrm{D}$ ridge created by the forces. While moving along the path, the user can set control points which are used to approximate the path with a spline. The advantage of this approach becomes apparent in situations where the image data is not sufficient to discriminate the object boundaries. At regions with unclear image information, a user can exert forces on the haptic device to leave the precalculated path by pushing the 3D cursor into the direction, where the path continues. The segmentation of tubular structures can be optimally facilitated, if all cross-sections of the object would be orthogonal to the centerline. Therefore, 
we use the spline, that is generated interactively to determine the appropriate orientation of a free slice plane through the data volume. The next step is to use an active contour model to extract the $2 \mathrm{D}$ cross-sections of our structure of interest. The adaptive reorientation of the cutting slice allows us to make a rough assumption about the shape of the cross-section, which should be approximately circular. Furthermore, as we always update the slice orientation, we can use the segmentation of the previous step as a fairly good initialization for the next cross-section. This can be done, because the deviation between subsequent crosssections in a tubular structure is usually small. To estimate the sensitivity of our approach and to understand its limitations, we performed a first case study as an initial proof of concept. As an area of high medical relevance, we selected virtual colonoscopy for the validation of our approach. The dataset used in our study, a $256 \times 256 \times 223$ CT scan of the human abdomen, was acquired at the Institute of Diagnostic Radiology of the Zurich University Hospital.

\section{$3 \quad$ First Results and Future Research}

We used our interactive system to create the centerline through the tubular structure and obtained the reformatted cross-sections along the path. The crosssections on each slice were extracted with an active contour model. Due to the reformatting of the cross-sections and the initialization propagating from slice to slice, this process could be done without any user interaction. Nevertheless, it has to be mentioned, that this processing step was eased by the good image quality of our dataset. Apart from the case study described in this paper, we also applied our system to the segmentation of the small intestines (A 256x256x147 CTdataset of the abdomen, provided by the Department of Radiology, Semmelweis University Budapest). While in the former case conventional algorithms could also be used successfully, they failed on the latter one due to the problems described above. In contrast to this, our approach succeeded on this complex dataset, too. Nevertheless, we have to mention that a decrease of image data quality was accompanied by increased user interaction time and effort. Further evaluation, especially for more complex data has to be done in our on-going project. Especially, the suitability of this approach for clinical practice has to be studied.

\section{References}

1. Dirk Bartz and Özlem Gürvit. Haptic navigation in volumetric datasets. In Proceedings of the Second PHANToM Users Research Symposium. Swiss Federal Institute of Technology, 2000.

2. Ken Hinckley. Haptic Issues for Virtual Manipulation. PhD thesis, School of Engineering and Applied Science, University of Virginia, 1996.

3. Serra L., Ng H., Chua B.C., and T. Poston. Interactive vessel tracing in volume data. In ACM 1997 Symposium on Interactive 3D Graphics, pages 131-137, 1997.

4. Steven O. Senger. User-directed segmentation of the visible human data sets in an immersive environment. In Richard A. Banvard, Prof. Francesco Pinciroli, and Pietro Cerveri, editors, The Second Visible Human Project Conference Proceedings, 1998. 